\title{
Inaction publique et immobilisme privé : quand le renouvellement urbain échoue à Philadelphie
}

\author{
Public inaction and private stasis: urban \\ redevelopment's failure in Philadelphia
}

\section{Florence Nussbaum}

Doctorante en géographie, université Paris-Diderot - UMR 8504 Géographie-Cités

Résumé L'attention portée aux villes de tradition industrielle aux États-Unis concerne avant tout les initiatives de renouvellement urbain. Or une large part des anciens quartiers industriels demeure en marge de cette dynamique et semble plutôt marquée par un délabrement durable. À partir de l'exemple de Kensington à Philadelphie, nous avons cherché à savoir si cette persistance du délaissement témoignait du désintérêt des principaux acteurs en présence ou, au contraire, révélait les stratégies de contrôle de l'espace mises en place par le secteur privé. Au terme de notre travail, il est apparu que la léthargie de ce quartier visible à travers le grand nombre de propriétés vacantes - découle à la fois de l'immobilisme calculé de certains acteurs privés et de l'impuissance des services municipaux. D'une part, la rétention foncière et la spéculation immobilière de nombreux propriétaires contribuent à entretenir une situation figée qui exclut toute rénovation. D'autre part, la municipalité est paralysée par un budget insuffisant et un manque d'organisation hérité de longue date, de sorte qu'elle ne parvient pas à limiter l'ampleur de la vacance. En définitive, I'abandon apparent de tels espaces résulte en réalité d'intérêts locaux contradictoires tant sur le plan politique que sur le plan économique.

Abstract US cities with an industrial past have mostly been studied through the scope of urban renewal initiatives. Yet, many former industrial neighborhoods do not participate in this dynamic and are increasingly affected by urban blight. Based on the case study of Kensington in Philadelphia (Pennsylvania), this paper aims at understanding the mechanisms accounting for this persistent abandonment. Far from revealing the indifference of the stakeholders in this neighborhood, this inertia indicates in fact that they developed strategies to control the area, therefore hindering any development. Indeed, the slump in this neighborhood-made visible through the large amount of vacant properties - results from the deliberate immobilism of private stakeholders as well as the powerlesslessness of municipal services. The former benefit from the legal framework whose protection enables them to retain properties and to speculate on land values while the action of the latter is hampered by a long-standing disarray and an insufficient budget. To conclude, as abandoned as they may seem, former industrial neighborhoods such as Kensington are in fact at the heart of the prevailing political and economic stakes for several players in the area.

Mots-clés Propriété, délabrement urbain, abandon, spéculation, quartier industriel, politique publique, gouvernance urbaine 
Keywords Property, blight, abandonment, speculation, industrial neighborhood, public policy, urban governance

Dans la nuit du 8 au 9 avril 2012, l'ancienne fabrique Thomas Buck qui dominait le quartier d'East Kensington à Philadelphie du haut de ses six étages fut ravagée par un incendie, aboutissant à l'effondrement de la structure et à la mort de deux sapeurs-pompiers. Abandonné durant plusieurs décennies, le bâtiment appartenait depuis 2009 à des investisseurs new-yorkais mais il n'avait fait l'objet d'aucune entreprise de rénovation ou du moins de sécurisation, au point que les riverains multipliaient les plaintes auprès de la Ville. En outre, il est apparu que les propriétaires devaient plus de 50000 dollars de taxes foncières impayées à la municipalité.

Cette catastrophe a relancé la polémique sur la gestion des bâtiments vacants et sur la responsabilité de leurs propriétaires. Selon un rapport de la Brookings Institution rendu en 2000, on comptait 36,5 structures abandonnées pour 1000 habitants à Philadelphie contre 2,6 en moyenne pour les 83 villes états-uniennes étudiées. Outre l'impact paysager négatif d'un bâti dégradé et de parcelles couvertes de détritus, les propriétés vacantes sont également associées à un risque accru d'incendie et à l'installation d'activités illégales (trafic, drogue, prostitution). Le sentiment d'insécurité qui en résulte, allié à un état de délabrement général, a tendance à rebuter les résidents potentiels et à dévaloriser l'image du quartier.

Les bâtiments vacants apparaissent alors comme les symptômes d'un délaissement généralisé. Mais cette inertie n'est pas tant le signe d'un désintérêt des principaux acteurs en présence qu'un indice révélant les stratégies de contrôle de l'espace mises en place par certains propriétaires privés, aboutissant à un maintien de l'état de dégradation du quartier. Ce phénomène est encore renforcé par l'impuissance des autorités locales, du fait de moyens insuffisants et des restrictions imposées par la législation.

\section{Faire une place aux territoires de la stase urbaine}

\subsection{Les propriétés vacantes au cœur des villes américaines}

L'inertie foncière et immobilière - caractérisée par des prix du foncier peu élevés et un fort taux de vacance - concerne principalement les villes états-uniennes touchées par une période de déclin démographique : suite à la phase de désindustrialisation des années 1970, ces villes ont perdu une large part de leur population, de sorte que le tissu urbain est aujourd'hui surdimensionné par rapport à la population résidente. Détroit constitue l'exemple paradigmatique de ces shrinking cities puisque sa population a été divisée par deux depuis 1970. Aujourd'hui, plus du tiers de son territoire municipal est inhabité (Popelard, 2009 ; Reese, 2006). Ce phénomène concerne également les autres villes de la Rust Belt comme Philadelphie dont la population a chuté de $22 \%$ entre 1970 
et 2000, la classant dans le groupe des severely declining cities in declining metropolitan areas (Bradbury, Downs et Small, 1982) avec quinze autres métropoles dont notamment Détroit, Buffalo, Pittsburgh et Cincinnati.

La question du délaissement urbain se pose de plus en plus dans de nombreuses villes américaines, même au sein de l'ancienne Sun Belt en croissance depuis les années 1960. À Houston (Texas) ou Phoenix (Arizona), certains quartiers centraux offrent le même spectacle de désolation que les anciens quartiers industriels de la Rust Belt : bâtiments abandonnés, terrains vagues et détritus dessinent un paysage que l'on retrouve désormais dans nombre de grandes villes des États-Unis. L'existence de ce phénomène dans des villes où le nombre d'habitants continue d'augmenter $(+29 \%$ à Houston et $+47 \%$ à Phoenix entre 1990 et 2010) suggère que le déclin démographique n'est pas la seule explication de ce processus de délaissement.

La crise immobilière qui a débuté en 2008 a contribué à accroître le nombre de propriétés vacantes car elle a conduit à la saisie de nombreuses propriétés en situation de défaut de paiement (foreclosure). D'après le bureau du recensement américain (US Census), le nombre de propriétés vacantes aux États-Unis a augmenté de $43 \%$ entre 2000 et 2010 , passant ainsi de 7 à 10 millions au cours de la décennie. Des États jusqu'ici très attractifs comme la Floride ou la Californie sont désormais concernés par ce phénomène : sur la même période, dix États ont connu une augmentation du nombre de propriétés vacantes supérieure à $70 \%$. Parmi ceux-ci, on trouve notamment le Nevada (126\%), l'Arizona (92\%) et la Floride (90 \%). La crise de 2008 a entraîné en particulier une nette augmentation du nombre de real-estate owned properties (REO), c'est-à-dire de propriétés saisies par un créancier - le plus souvent une banque - pour rembourser la dette de leur ancien propriétaire. Dans le contexte de la crise immobilière, nombre de ces propriétés sont restées invendues et les banques attendent le moment propice pour les remettre en vente, contribuant à l'inflation du nombre de propriétés vacantes.

À l'échelle intra-urbaine, les territoires les plus touchés par le délaissement sont ceux qui abritaient les travailleurs de l'industrie et, plus récemment, les propriétaires les plus fragiles économiquement qui ont été les premiers chassés par la crise des subprimes. Dans les villes états-uniennes, ces espaces de transition correspondent à une zone intermédiaire, entre les suburbs anciennement attractives et le downtown, revitalisé progressivement depuis les années 1960 par la multiplication des complexes commerciaux (Defilippis, 1997) et des immeubles de bureaux (Beauregard, 2005).

\subsection{Au-delà de la division centre-périphérie, des trajectoires de quartiers très diverses}

Le modèle urbain appliqué aux États-Unis opposait traditionnellement la pauvreté de la ville-centre (inner-city) à l'opulence des suburbs (Beauregard, 2002). Le départ des classes moyennes - blanches en particulier - avait en effet participé à l'émergence d'une «nouvelle pauvreté urbaine » piégée dans des ghettos sans 
perspective d'ascension sociale (Wilson, 1997), tandis que de nouveaux centres attractifs se formaient en périphérie des grandes métropoles (Garreau, 1992). La politique d'urban renewal, caractérisée par la construction massive de logements, de tours de bureaux et d'infrastructures reliant le centre-ville aux banlieues, et visant à ramener les classes moyennes blanches dans le centre (Avila et Rose, 2009 ; Rast, 2001), a rendu au downtown son importance symbolique, politique et financière, notamment grâce à la constitution de central business districts (Doussard, Peck et Theodore, 2009 ; Rast, 2001).

Cependant, entre le downtown revalorisé et les surburbs attractives se trouvent différents espaces très hétérogènes, certains bénéficiant largement de la croissance économique du centre-ville à travers l'expansion progressive du processus de gentrification, tandis que d'autres semblent abandonnés par les acteurs traditionnels du développement économique (Wyly et Hammel, 1999). Parmi ces espaces en marge de l'investissement privé, on retrouve notamment la couronne industrielle du siècle dernier. Dépeuplée par la désindustrialisation, elle concentre aujourd'hui la part la plus pauvre de la population urbaine et les minorités raciales. C'est d'ailleurs principalement à travers la question raciale que cette zone a longtemps été étudiée, et ce dès les années 1920 avec les sociologues de l'École de Chicago, puis plus généralement via l'étude de la très grande pauvreté dont ont émergé les notions d'underclass et de ghetto (Massey et Denton, 1993 ; Wilson, 1997). Plus récemment, l'attention s'est portée sur un autre élément au sein de ces espaces intermédiaires : les first-tier suburbs (Vicino, 2008) ou inner-ring suburbs (Hanlon, 2008, 2009), c'est-à-dire les implantations périurbaines les plus anciennes, en bordure immédiate de la ville-centre, caractérisées par un bâti vieillissant, un déclin démographique et une paupérisation croissante (Lee et Leigh, 2007). Les analyses insistent aujourd'hui sur cette diversité des espaces de l'entre-deux et poussent donc à sortir d'une dichotomie réductrice qui opposerait ville-centre en déclin et suburbs florissantes (Hanlon, Short et Vicino, 2009), d'autant que la situation socio-économique des quartiers est soumise à une forte variabilité temporelle, dessinant des trajectoires ascendantes et descendantes complexes au sein de l'aire urbaine (Mikelbank, 2011).

\subsection{D'une géographie de la variation à une géographie de l'inertie}

De fait, la ville américaine est généralement pensée en termes de dynamiques, suggérant que les territoires suivent nécessairement une courbe ascendante (croissance démographique, valorisation économique) ou descendante (fuite des habitants, désinvestissement). Dans le cas des anciens quartiers industriels, l'importance du processus de gentrification a attiré l'attention sur les phénomènes de valorisation et a suscité de nombreuses controverses sur leur interprétation. Les penseurs néo-marxistes y voient d'abord le signe d'un retour au centre du capital et non des hommes (Smith, 1979) sous l'effet du spatial fix (Harvey, 1985), c'est-à-dire la recherche d'un nouvel ancrage spatial du capital afin de compenser la sur-accumulation engendrée par le système capitaliste. Les théoriciens de la demande expliquent au contraire cette valorisation par des choix résidentiels 
individuels (Berry, 1985), ceux d'une « nouvelle classe culturelle » (Ley, 1996) en recherche d'authenticité (Zukin, 2009) qui suscite le réinvestissement immobilier des quartiers anciens. Ces études permettent d'analyser les paramètres qui expliquent l'évolution d'un quartier, en l'occurrence vers une valorisation foncière et immobilière et l'attraction de nouvelles populations.

De la même façon, l'étude du déclin de certains quartiers a permis d'éclairer les processus à l'œuvre et les acteurs intervenant dans le délaissement progressif des territoires. Il est ainsi apparu que le déclin n'était pas un phénomène "naturel » qui résulterait mécaniquement des fluctuations du marché, mais bien plutôt la conséquence logique des stratégies déployées par divers acteurs publics et privés tirant parti des conditions offertes par le contexte économique et politique (Aalbers, 2006 ; Charney, 2001).

C'est le cas notamment du redlining, utilisé entre 1934 et 1968 aux ÉtatsUnis, qui consistait en la délimitation par les banques de zones dans lesquelles elles n'accordaient pas de crédit immobilier, ou seulement sous des conditions désavantageuses comme un taux d'intérêt prohibitif (Aalbers, 2006 ; Hillier, 2003). Cette pratique discriminatoire a eu pour effet de renforcer la ghettoïsation des populations noires en leur refusant l'accès à la propriété. Les foyers afroaméricains étant exclus de l'offre de crédit traditionnelle, ils devaient recourir aux services d'agents immobiliers qui proposaient le paiement de leurs propriétés par mensualités, via des land installment contracts. En plus d'aboutir à un prix de vente exorbitant, ces contrats dissimulaient des clauses permettant l'expulsion des acheteurs au moindre retard de paiement, quelles que soient les sommes déjà versées, de sorte que les foyers victimes de telles opérations immobilières perdaient à la fois la propriété de leur maison et l'épargne accumulée durant des années pour rembourser leur «crédit» (Satter, 2010). La stratégie appelée blockbusting a également contribué au déclin de certains quartiers dans les villes américaines (Orser, 1994 ; Seligman, 2005) : en faisant croire à l'installation de familles noires, certains agents immobiliers incitaient les résidents des quartiers blancs à vendre leur logement à bas prix par peur d'une dévalorisation soudaine de l'immobilier. Ils vendaient ensuite ces propriétés à des familles noires avec les conditions défavorables déjà évoquées, aboutissant à une paupérisation croissante du quartier et à la dégradation du bâti.

Ces pratiques montrent à la fois la dimension foncière et immobilière du processus de déclin, et le rôle joué par une nébuleuse d'acteurs, privés mais aussi publics (Kimble, 2007), dans la relégation des populations les plus vulnérables dans des quartiers de plus en plus dégradés (Gotham, 2002). Si ces phénomènes sont étroitement liés aux questions de ségrégation raciale dominantes à l'époque, les analyses récentes sur la crise des subprimes de 2008 ont montré la persistance de pratiques prédatrices, concernant en particulier l'accession à la propriété des ménages modestes (Saegert, Fields et Libman, 2009 ; Wyly, Atia, Foxcroft, Hamme et Phillips-Watts, 2006), au point que l'octroi préférentiel de prêts subprime aux populations les plus défavorisées, le plus souvent noires, a pu être qualifié de reverse redlining (Squires, 2005). 
Les cadres explicatifs fournis par l'analyse des processus de déclin ou au contraire de valorisation permettent de mieux comprendre le fonctionnement des espaces de l'entre-deux évoqués plus haut et les « effets de quartier » éventuels (Ham, Manley, Bailey, Simpson, et Maclennan, 2012). Cependant, le quartier de Kensington à Philadelphie ne semble s'inscrire dans aucune de ces deux tendances : avec une population qui stagne autour de 15000 habitants depuis 1990, un taux de vacance autour de $12 \%$ qui a légèrement baissé tout en restant au-dessus de celui de Philadelphie, et un marché immobilier atone ${ }^{1}$, Kensington apparaît plutôt comme un quartier peu attractif mais relativement stable. Cette inertie, visible en particulier dans le maintien d'un grand nombre de terrains vagues et de bâtiments inoccupés, n'est peut-être qu'un stade transitoire avant une phase de gentrification ou un déclin plus prononcé. Mais cet état en luimême n'a rien d'évident : la zone en périphérie du centre-ville où se trouve Kensington compte en effet à la fois des quartiers qui ont pris de la valeur (Fishtown, Northern Liberties) et des quartiers en déclin (Feltonville, North Philadelphia). Dès lors, comment expliquer que ce territoire ne soit pas gagné par les dynamiques voisines?

La situation de ce quartier nous interroge d'autant plus qu'il ne représente pas une exception dans la réalité urbaine contemporaine aux États-Unis. Les quartiers de Woodlawn à Chicago ou Fifth Ward à Houston présentent des caractéristiques assez similaires du point de vue de l'ampleur de la vacance et de l'inertie démographique et immobilière.

\section{Kensington, un ancien quartier industriel délaissé}

\subsection{Cadre méthodologique : une approche par la propriété}

Pour tenter d'éclairer les déterminants de la stase observée à Kensington, nous avons choisi de nous intéresser à la structure de la propriété, notamment des propriétés vacantes qui sont le signe le plus marquant de cette inertie. Ce choix repose d'une part sur l'importance de la vacance comme caractéristique intrinsèque à ces quartiers, et d'autre part sur la volonté d'explorer des facteurs explicatifs complémentaires des mouvements de capitaux et des stratégies résidentielles pour rendre compte des dynamiques urbaines récentes.

Il importe d'abord de distinguer les bâtiments " abandonnés », c'est-à-dire ceux pour lesquels le propriétaire ne paie plus de taxes, et les bâtiments "vacants ", pour lesquels le propriétaire continue à payer les taxes réglementaires à la ville (Kraut, 1999). Les villes n'ont de pouvoir que sur les bâtiments « abandonnés » (tax-delinquent properties), qu'elles peuvent saisir pour réclamer les taxes qui

1 Selon le site Trulia.com qui répertorie les annonces immobilières aux Etats-Unis, le prix de vente médian pour les biens immobiliers résidentiels à Kensington était de 108750 dollars fin 2014, soit un prix inférieur de 21,76 \% au prix médian pour la ville de Philadelphie. En outre, le prix de vente médian aurait connu une baisse de $11 \%$ au cours des cinq dernières années. 
leur sont dues. Elles ne peuvent intervenir au sujet des bâtiments vacants que s'ils constituent un danger pour la population, et ce au prix de procédures longues et coûteuses. De plus, si le recensement américain offre des données sur les propriétés vacantes, il n'existe pas de liste des propriétés abandonnées: pour obtenir des informations sur celles-ci, il faut consulter la fiche individuelle de chaque propriété sur le site de l'Office of Property Assessment (OPA) de Philadelphie.

Nous avons ensuite tracé le cadre réglementaire dans lequel se déroulent toutes les relations de propriété à travers l'étude des lois en vigueur à différentes échelles, de la ville de Philadelphie ${ }^{2}$ à l'État de Pennsylvanie ${ }^{3}$. Nous avons également essayé d'établir précisément le rôle des différentes instances intervenant dans la gestion de la propriété foncière à Philadelphie, depuis l'inspection des bâtiments jusqu'à leur saisie éventuelle en cas de manquement grave du propriétaire à la législation, en nous appuyant sur les sites internet des instances concernées ainsi que sur plusieurs rapports analysant les dysfonctionnements des agences municipales de Philadelphie vis-à-vis des propriétés vacantes (Econsult Corporation, 2010 ; Philadelphia Local Initiatives Support Corporation, 2009). Nous avons complété ces informations par des entretiens menés entre janvier et mai 2012 auprès de différents représentants des institutions concernées par la gestion des propriétés vacantes ou abandonnées : une personne ayant exercé des responsabilités à la Philadelphia Redevelopment Authority (PRA) jusqu'en 2010 $0^{4}$; un ancien directeur de l'Office of Housing and Community Development (OHCD); et une personne chargée de la mise en ouvre du Neighborhood Stabilization Program (NSP) à la Redevelopment Authority. Les questions posées lors de ces entretiens portaient sur le rôle précis joué par chacune de ces instances, les modalités pratiques de leur intervention (temporalité, difficultés éventuelles), et la politique de la municipalité à l'égard des propriétés vacantes et de leurs propriétaires.

D'autre part, l'analyse de la structure de la propriété passe par l'étude de la situation actuelle de certaines parcelles. Pour ce faire, nous disposions principalement de la base de données du Cartographic Modeling Laboratory de l'université de Pennsylvanie : le Neighborhood Information System ${ }^{5}$. Celui-ci propose une base de données à l'échelle de la parcelle, permettant de connaître pour chacune le nom du propriétaire et son adresse éventuelle, la date et le prix d'acquisition de la propriété, le zonage pour la parcelle, sa situation fiscale en 2007, les violations éventuelles du code de la propriété constatées par le service

2 The Philadelphia Code, disponible en ligne à l'adresse: http://www.amlegal.com/nxt/gateway. $\mathrm{dII} /$ Pennsylvania/philadelphia_pa/thephiladelphiacode? $\mathrm{f}=$ templates $\$ \mathrm{fn}=$ default.htm $\$ 3.0 \$ \mathrm{vid}=$ amlegal:philadelphia_pa.

3 Consolidated Statutes of Pennsy/vania : http://www.legis.state.pa.us/cfdocs/legis/LI/Public/cons_index.cfm. The Pennsy/vania Code : http://www.pacode.com/index.html.

4 De façon à préserver l'anonymat de nos interlocuteurs, les informations données sur leur fonction exacte sont volontairement assez vagues.

5 Disponible sur le site du Cartographic Modeling Lab : http://www.cml.upenn.edu/nis/. 
des Licenses and Inspections (L \& I) jusqu'en 2005, le statut « vacant » ou non de la parcelle en 2000, ainsi que toute déconnexion du réseau d'eau (jusqu'en 2007) ou de gaz (jusqu'en 1999). La quantité de données disponibles est donc conséquente mais leur usage reste délicat dans la mesure où les dates varient pour chaque type de donnée considéré. Bien plus, l'exploitation de ces informations est laborieuse car leur traitement informatique est impossible, ce qui empêche une cartographie exhaustive à l'échelle du quartier dans le temps imparti, d'autant qu'il existe de nombreuses incohérences entre les données de l'Office of Property Assessment (OPA), pour les informations relatives au propriétaire, et celui du Department of Revenue, pour la situation fiscale de la propriété.

Finalement, afin de déterminer les relations entre les différents partis en présence et d'ébaucher leurs stratégies concernant la propriété foncière sur notre terrain d'étude, nous avons réalisé quelques entretiens avec des acteurs locaux : deux promoteurs et un agent immobilier investis dans divers projets de construction ou de réhabilitation dans le quartier, la directrice de la New Kensington Community Development Corporation (NKCDC), organisation à but non lucratif chargée du développement local, et le président d'une association de quartier. Ces entretiens portaient sur l'image du quartier pour chacun de ces groupes, leur connaissance des procédures d'acquisition des propriétés vacantes, leur opinion vis-à-vis de la gestion locale de la vacance et leurs aspirations en termes de propriété foncière et immobilière. Nous avons également assisté entre les mois de mars et mai 2012 à plusieurs réunions des trois associations de quartier qui se partagent Kensington : l'EKNA (East Kensington Neighbors Association), la FNA (Fishtown Neighbors Association) et l'ORCA (Old Richmond Civic Association) de façon à avoir un aperçu des préoccupations des résidents - du moins de ceux qui participaient à ces réunions.

\subsection{Inertie d'un quartier délaissé}

Kensington constitue un cas typique des espaces de l'entre-deux évoqués plus haut. Qualifié de «mine d'or » dans la presse locale en 1929, il était considéré comme l'un des quartiers les plus attractifs durant l'âge d'or industriel. En 1951, un journal local le décrit ainsi : Many of its industries are known and their products sold throughout the world. The industries employ thousands and are unquestionably the biceps of the city's industrial might (The Sunday Bulletin, 12 août 1951). Ce passage témoigne de l'importance symbolique de Kensington, qui apparaissait non seulement comme le moteur économique de Philadelphie, mais également comme une vitrine pour la ville à l'échelle mondiale. À partir des années 1960, le déclin de l'activité industrielle et la hausse du chômage ont suscité le départ d'une partie de la population du quartier vers la périphérie ou les villes du Sud en croissance, entraînant l'abandon d'une large part du patrimoine bâti, tant industriel que résidentiel. Aujourd'hui, le quartier est encore très marqué par l'impact de la désindustrialisation et il est aussi célèbre pour sa réputation de drug corner de Philadelphie que pour son passé productif au cour du workshop of the world qu'était la ville. 
Kensington, un quartier de Philadelphie proche du centre et bien desservi

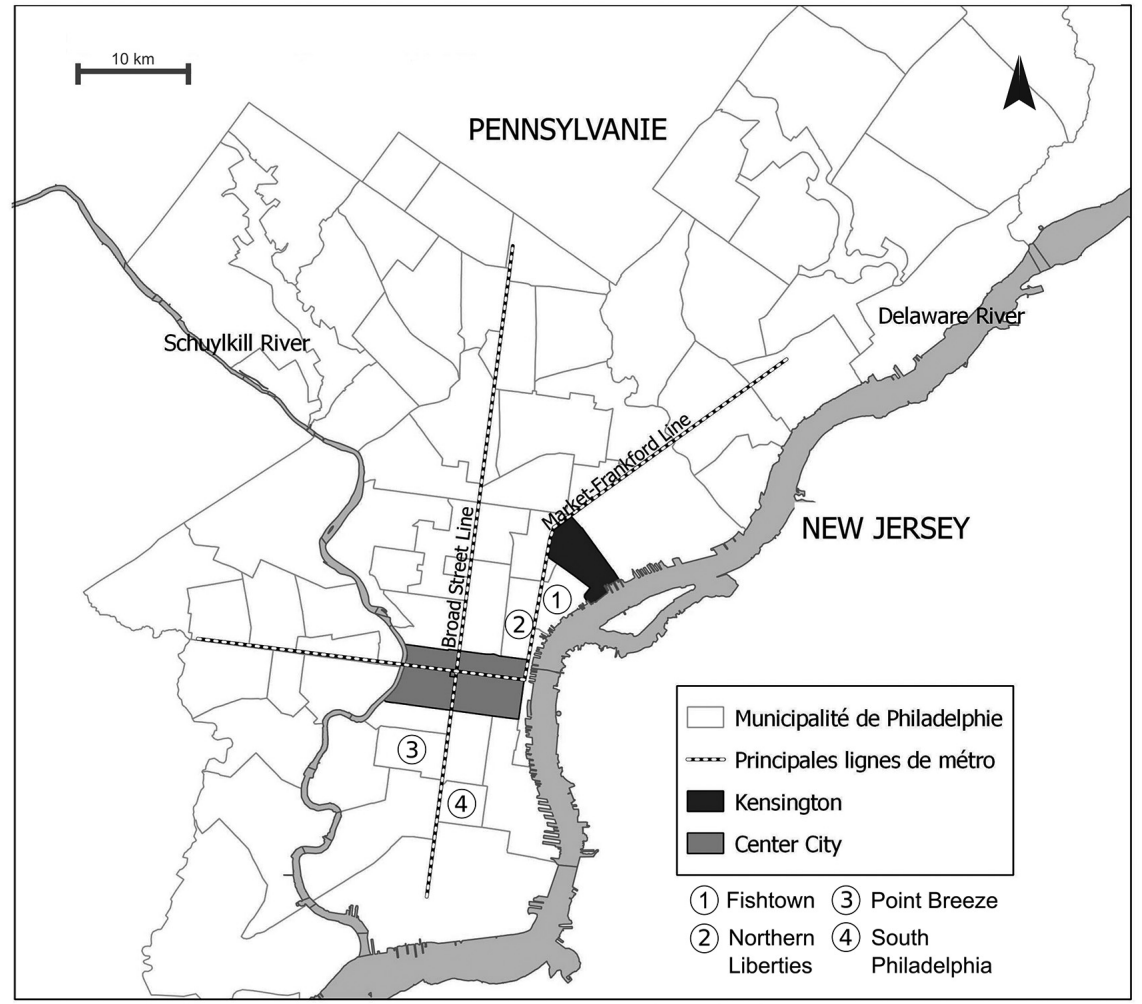

F. Nussbaum, 2013. Source : Neighborhood Information System (University of Pennsylvania)

Fig. 1 Carte de situation de Kensington au sein des limites municipales de Philadelphie Location of Kensington within Philadelphia's municipal borders

Bien qu'il soit situé à quinze minutes du City Hall par la ligne de métro aérien Market-Frankford (Figure 1), le quartier n'a bénéficié d'aucun projet de revitalisation particulier, les efforts de la municipalité s'étant concentrés sur l'hypercentre de la ville, redevenu attractif suite à la politique de downtown redevelopment menée dans les années 1990. Selon l'ancien directeur de l'OHCD (Office of Housing and Community Development) que nous avons interviewé, when you go downtown in the vicinity of City Hall, everything that is now a hotel, or a condo, or a luxury apartment building, in 1995 was vacant or substantially vacant. Every one of those buildings. Depuis la réhabilitation du centre-ville, l'intérêt des acteurs privés s'est peu à peu déplacé vers la périphérie, en bordure immédiate de Center City : South Philadelphia et Northern Liberties (nord-est) au début des années 2000, puis Point Breeze au sud et Fishtown au nord au milieu des années 2000. Mais l'étude de la carte indiquant les propriétés dont la valeur est la plus faible sur le marché montre la persistance d'une zone en crise en bordure 
immédiate du downtown (Figure 2). Alors que le centre-ville et les zones les plus périphériques comptent très peu de propriétés aussi bon marché, le downtown est entouré, en particulier au nord et à l'ouest, par des territoires où la part des propriétés dévalorisées est nettement supérieure au reste de la municipalité, dépassant parfois $20 \%$ des résidences principales.

\section{Une concentration des propriétés fortement dévalorisées au nord du centre-ville de Philadelphie}

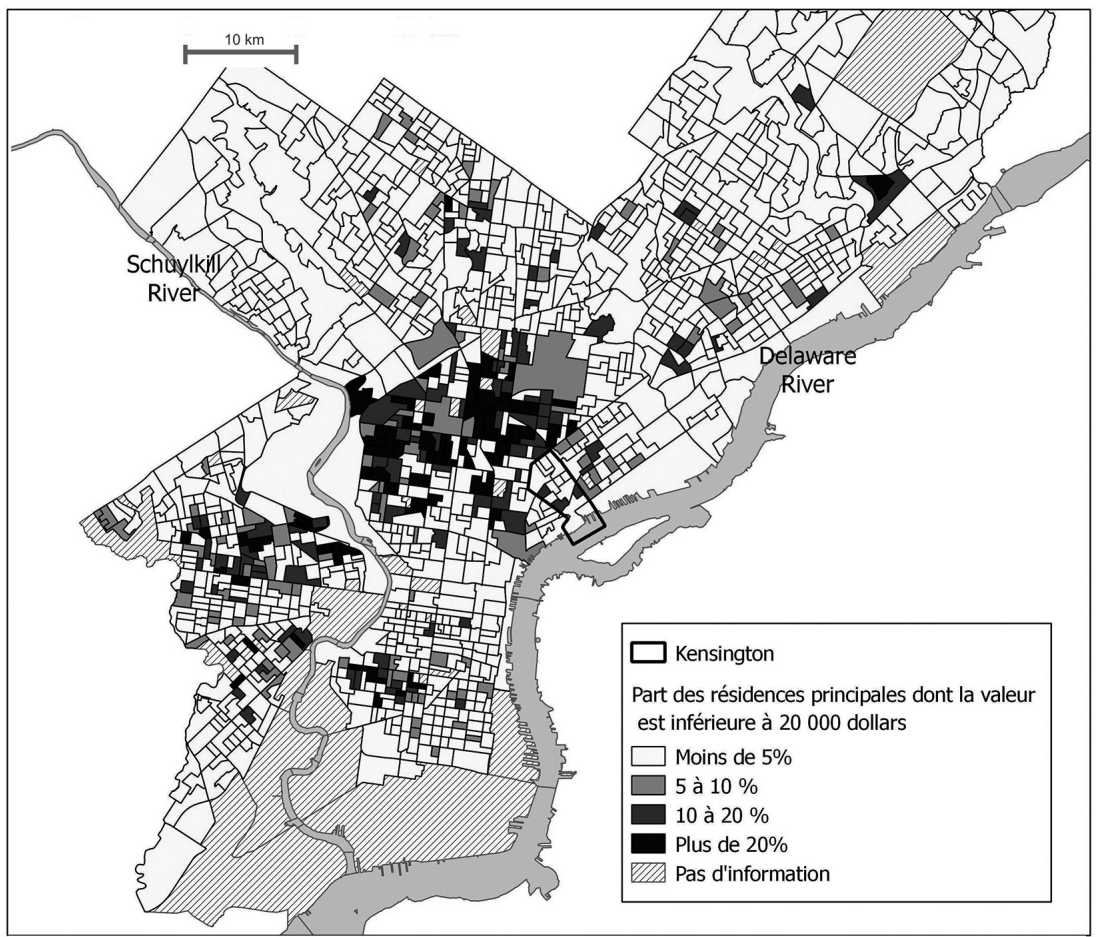

F. Nussbaum, 2013. Sources : Neighborhood Information System (U. of Pennsylvania), US Census (2012).

Fig. 2 Répartition des propriétés dont la valeur est la plus faible sur le marché immobilier de Philadelphie.

Location of the least valuable properties in Philadelphia's real estate market

Kensington se situe au sud-est de cette zone en crise et ne semble pas aussi marqué par le processus de dévalorisation. Mais avec 32,3\% de la population sous le seuil de pauvreté alors que la moyenne pour la ville est de $22,1 \%$, Kensington apparaît néanmoins comme un quartier en difficulté. L'héritage industriel y est encore très présent puisque la part du secteur résidentiel n'est que de $74 \%$ contre près de $80 \%$ pour la ville de Philadelphie ${ }^{6}$, ce qui pose la

6 Données de 2007. Source : Neighborhood Base, University of Pennsylvania. 
question du devenir du bâti industriel, parfois difficile à réhabiliter mais coûteux à détruire. Le quartier a gardé de son passé ouvrier une population majoritairement blanche : en 2010, les Blancs - Hispaniques compris - constituent encore $71 \%$ de la population de Kensington alors que ce taux tombe à $41 \%$ pour la ville entière $^{7}$. Les minorités ethniques sont également représentées mais la part de la population Afro-Américaine $(7,4 \%)$ est nettement inférieure à la moyenne municipale $(43,4 \%)$.

L'inertie qui caractérise le quartier est particulièrement visible dans le paysage : terrains vagues, vitres brisées, portes condamnées, graffitis ou décharges sauvages sont autant de signes de l'abandon du territoire. La photographie présentée en figure 3 en témoigne : au premier plan se trouve une friche laissée par l'incendie d'une ancienne usine il y a plusieurs années; sur la droite, un rang incomplet de rowhouses ${ }^{8}$ laisse voir les espaces laissés par les maisons démolies; et à gauche l'ancienne bonneterie Thomas Buck est condamnée mais mal sécurisée, ce qui peut expliquer son incendie moins d'un mois après la prise de vue.

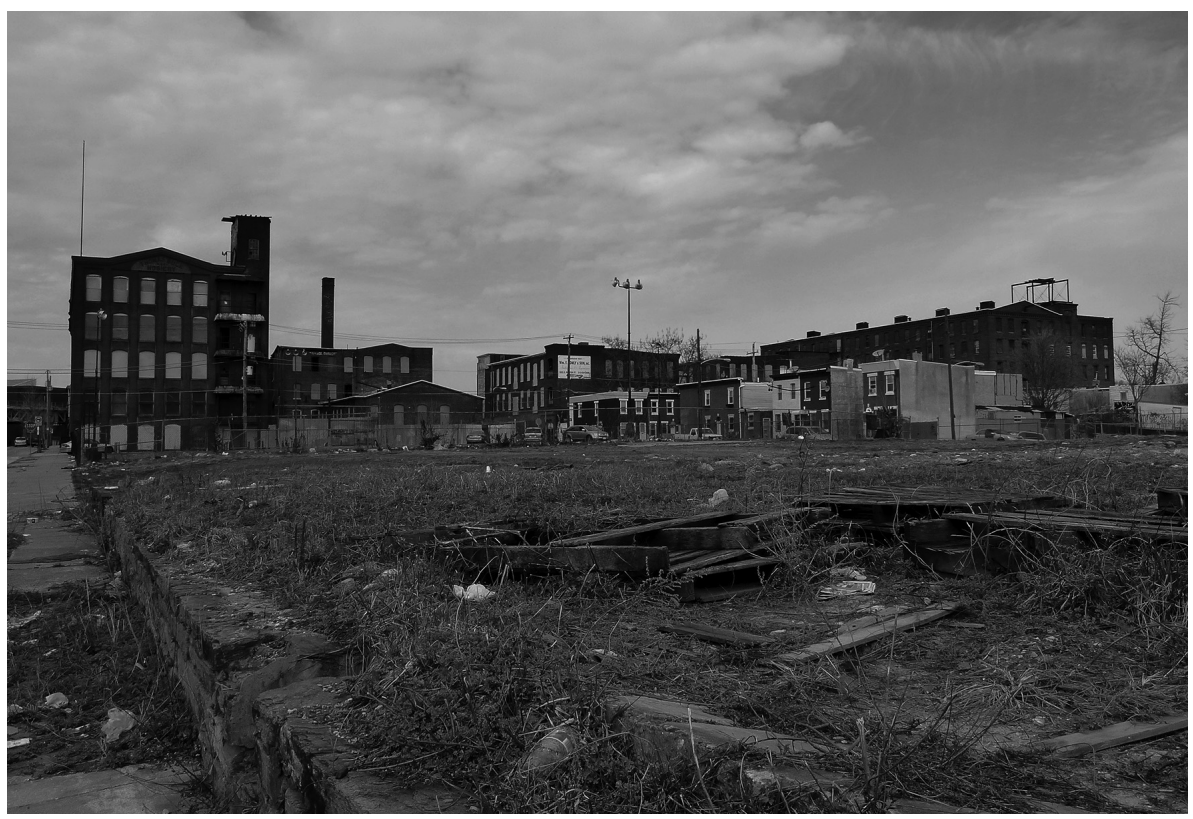

Cliché : F. Nussbaum, 12 mars 2012.

Fig. 3 Le paysage de Kensington, vu depuis I'angle de York Street et Emerald Street en direction du nord-ouest.

The landscape of Kensington, as seen from the corner of York Street \& Emerald Street facing northwest.

7 Source pour toutes les données sur la composition de la population : US Census, 2010.

8 Maisons mitoyennes typiques de I'habitat ouvrier nord-américain. 


\section{Pesanteurs juridiques et stratégies individuelles des propriétaires : I'inaction dans le quartier de Kensington}

\subsection{L'inertie du foncier, conséquence des stratégies de contrôle de l'espace par les acteurs privés}

\subsubsection{Des investissements privés limités}

La bordure sud-ouest de Kensington marque la frontière avec le quartier gentrifié de Fishtown, devenu très attractif depuis les années 1990. Pourtant, la dynamique d'investissement ne gagne pas Kensington, et la quasi-totalité des maisons neuves construites dans le quartier depuis une dizaine d'années se situe à l'ouest de York Street, qui marque la limite entre la portion du quartier assimilée à Fishtown (avec qui elle partage l'association de résidents) et celle considérée comme appartenant véritablement à Kensington. Quelques nouvelles constructions sont visibles au sud-est, mais le nord ne compte aucune maison neuve.

Cela s'explique tout d'abord par le type de terrain disponible : les parcelles réservées à un usage résidentiel dans le Zoning Code sont les plus recherchées par les promoteurs car la demande est forte et la plus-value est importante. Or la partie nord du quartier, appelée East Kensington, comporte un grand nombre de parcelles réservées à un usage industriel, ce qui impose à l'acheteur de demander une dérogation au Zoning Code s'il veut y construire des logements et ajoute donc des délais supplémentaires au projet. En outre, les promoteurs sont sensibles à la "théorie de la vitre brisée » (Wilson et Kelling, 1982) : ils investissent plus volontiers dans un quartier dont l'état général est bon et se méfient des zones déjà délabrées par peur d'une dégradation continue du bâti. Ainsi, les parcelles vacantes aménagées en priorité sont celles situées dans des blocks majoritairement bâtis : la maison neuve vient combler un interstice laissé vide dans le rang de rowhouses, faisant disparaittre peu à peu les friches laissées par les maisons détruites. C'est le cas dans la zone sud à la frontière avec Fishtown, mais aussi à l'est d'Aramingo Avenue, en bordure de l'autoroute I-95 et du fleuve Delaware. Au contraire, lorsque les parcelles vides dominent dans un block, entourant quelques rares maisons comme c'est le cas entre Jasper Street et Emerald Street, l'investissement est trop risqué car l'ampleur de la vacance dévalue l'ensemble du pâté de maisons.

En second lieu, l'image du quartier joue un rôle important dans la vente d'une propriété et Kensington souffre encore de sa réputation passée. Un architecte du quartier peine à trouver des clients pour l'usine qu'il a réhabilitée en lofts, à la fois parce que les maisons individuelles ont plus de succès que les appartements, et parce que la connotation négative de " $K \&$ \& $A$ ", haut lieu du crime et de la drogue, reste attachée à Kensington. L'intersection des avenues Kensington \& Allegheny, qui se situe environ 800 mètres au nord de notre quartier d'étude par Kensington Avenue, est connue pour être le drug corner de Philadelphie depuis les années 1980, notamment sous l'impulsion du $K$ \& $A$ gang. In Philly, the word "Kensington" means crime, drugs and poverty. In other words: trash 
(Philadelphia Weekly, 20 avril 2011). À cela s'ajoute la psychose déclenchée à l'hiver 2010 par le Kensington strangler, responsable du meurtre de trois jeunes femmes dans le quartier. Fishtown n'est pas concerné par ce type de réputation et a bénéficié depuis plusieurs années de son image résidentielle et familiale, ainsi que de la montée des prix de l'immobilier. Dès lors, la zone située au sud-ouest de Kensington est nettement plus attractive car elle dépend de la Fishtown Neighbors Association (FNA) et, du point de vue des représentations, elle fait partie de Fishtown (Figure 4).

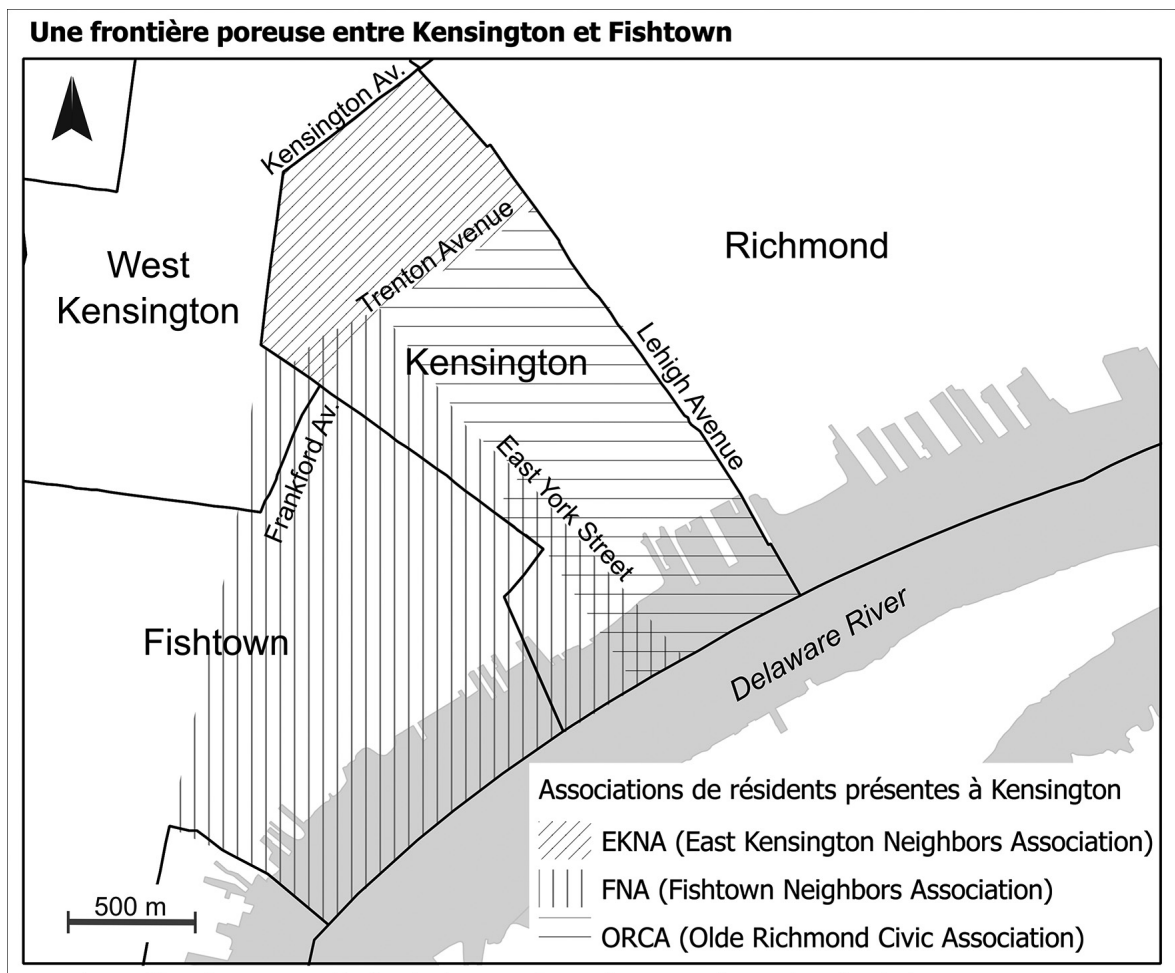

F. Nussbaum, 2015. Sources : Neighborhood Information System (University of Pennsylvania) ; nkcdc.org

Fig. 4 Les différentes associations de quartier qui se partagent Kensington Several civic associations operate in Kensington

\subsubsection{Une spéculation fondée sur la rétention foncière}

L'inertie du quartier s'explique également par l'importance de la spéculation foncière et immobilière. Une première forme de spéculation existe depuis longtemps dans les quartiers qui, comme Kensington, ont connu une phase de délaissement après la Seconde Guerre mondiale. Il s'agit des grands propriétaires qui ont constitué des empires immobiliers en achetant à très bas prix de nombreuses 
propriétés. Ceux-ci sont généralement qualifiés de slumlords (contraction de slum landlords: propriétaires de taudis), du fait de l'état de dégradation dans lequel se trouvent leurs propriétés. Cela répond à leur objectif de minimisation des dépenses afin d'accroitre leur profit: They maximise rent income from the property whilst disinvesting by avoiding expenditure upon it such as maintenance, property taxes, mortgage payments, utility charges etc. Finally when no rent can be obtained they abandon it (Karn et al., 1985, p. 122, cité par Aalbers, 2006). Dès lors, l'activité immobilière de Fishtown a paradoxalement pour effet de paralyser d'autant plus la situation à Kensington car les propriétaires n'abandonnent pas les propriétés les plus dégradées mais attendent de bénéficier de la montée des prix pour vendre leur bien. Ainsi, dans les zones comme Kensington où les prix sont très bas, les spéculateurs acquièrent la plupart des propriétés disponibles et refusent de vendre tant qu'ils ne peuvent obtenir un prix suffisamment élevé. Un article du Philadelphia City Paper (9 juin 2010) évoque l'exemple d'une maison délabrée possédée par le slumlord Robert Coyle. Lorsque le nouveau propriétaire de la rowhouse voisine a cherché à acquérir cette maison vacante en 2007, R. Coyle a repoussé l'offre à moins que l'acheteur ne lui propose 40000 dollars, pour une propriété qu'il avait achetée pour un montant de 8500 dollars en 1998. Le prix demandé était trop élevé pour l'acheteur potentiel, de sorte que la vente n'a pas eu lieu et la propriété est restée vacante.

Ces pratiques spéculatives s'appuient en particulier sur le vivier de propriétés à bas prix offertes par les Sheriff's Sales, durant lesquelles sont vendues aux enchères les propriétés qui ont été saisies par les pouvoirs publics parce que des dettes y étaient attachées. L'étude des registres de l'Office of Property Assessment (OPA) rend compte de ce phénomène : dans le block 1900 de Cumberland Street, au nord de Kensington, une société appelée " Great Philly Homes LLC » possède sept parcelles vacantes. La première des sept propriétés a été acquise en 2006, une seconde en février 2008, et les cinq autres ont toutes été achetées le même jour, le 3 mars 2008, à raison de 5200 à 11200 dollars par propriété. Cet achat multiple de propriétés suggère que le propriétaire a participé à une vente du Sheriff's Office. La société "Great Philly Homes LLC » possédait un terrain dans ce block de Cumberland Street depuis 2006 et l'acquisition de nouvelles parcelles vacantes lui assure un profit plus important en cas de valorisation de la zone car elle pourra proposer aux promoteurs des regroupements de parcelles pour un prix de vente supérieur. En effet, sur le côté impair de la rue, la société possède désormais quatre parcelles presque contiguës, entre lesquelles s'intercalent des propriétés qu'il lui sera sans doute possible d'acquérir. Tout d'abord, le 1935 East Cumberland Street est en défaut de paiement pour la taxe foncière depuis 1978, portant sa dette à un total de 5058 dollars selon le Revenue Department. "Great Philly Homes LLC » peut donc demander qu'elle soit saisie par le Sheriff's Office et mise aux enchères à hauteur des taxes dues à la Ville. D'autre part, le 1929 East Cumberland Street dépend du Department of Public Property qui, comme les autres agences publiques, n'a pas les moyens d'entretenir un patrimoine foncier trop important et cherche plutôt à confier cette charge à des acteurs privés. 
La propriété la plus délicate à obtenir sera le 1931 East Cumberland Street car elle appartient à la New Kensington Community Development Corporation (NKCDC), qui est peu susceptible de vendre dans la mesure où elle cherche plutôt à acquérir davantage de propriétés dans le quartier afin d'éviter leur dégradation. Néanmoins, si la NKCDC approuve le projet d'aménagement proposé sur ce regroupement de parcelles et si la vente de cette propriété lui octroie des fonds supplémentaires pour ses autres projets en cours, il est possible qu'elle accepte. "Great Philly Homes LLC » serait par conséquent propriétaire de sept parcelles contiguës, permettant de construire d'un bloc plusieurs maisons de ville neuves et de réaliser une plus-value considérable.

Les ventes du Sheriff's Office sont d'autant plus intéressantes pour les spéculateurs que la pratique de l'enchère fait apparaître les propriétés convoitées par d'autres investisseurs potentiels. D'après un rapport de la Local Initiatives Support Corporation (LISC), les agents municipaux ont constaté qu'une trentaine d'investisseurs privés achetaient de façon systématique des propriétés aux enchères organisées par le Sheriff : Sometimes they bid on a property while doing little research beforehand simply because they see that a $C D C^{9}$ or other community developer is bidding on it. This scenario became common during the strong real estate market from 2000-2006 (LISC, 2009, p 13).

Mais au-delà des pratiques de quelques spéculateurs, nombre de propriétaires ont recours à la rétention foncière à Kensington. En effet, la perspective d'une possible augmentation des prix si le phénomène de gentrification continue à progresser depuis le centre de Philadelphie incite les propriétaires à conserver leur bien dans l'attente d'une hypothétique valorisation. Plus encore, cela dissuade d'entreprendre des travaux de rénovation car, selon un agent immobilier interrogé sur place, le risque pour un propriétaire qui réhabilite son bien est de provoquer une hausse du prix des maisons et parcelles alentours, et ainsi d'assumer seul une dépense qui profite à tous. Cette logique contribue par conséquent au phénomène de rétention foncière car ceux qui possèdent une parcelle ou un bâtiment préfèrent souvent conserver leur bien en attendant qu'il prenne de la valeur sous l'effet des rénovations et constructions du voisinage, plutôt que de risquer une vente peu lucrative ou des travaux coûteux pour un profit modéré.

\subsubsection{Profiter des vides juridiques}

Enfin, la stase du quartier peut s'expliquer par le fait que certains propriétaires de bâtiments dégradés utilisent les failles de la législation en vigueur pour laisser leurs biens à l'abandon - et ainsi éviter de payer des taxes sur des propriétés dormantes. La loi spécifie en effet que toute intervention de la Ville doit être signalée au propriétaire sous la forme d'une notification - de violation d'un code municipal, de démolition, de saisie, etc. - qui doit à la fois être affichée de manière visible

9 Community Development Corporation (CDC) : organisation à but non lucratif, financée en grande partie par des fonds publics, ayant pour objectif I'amélioration des conditions de vie dans un quartier. 
sur la propriété concernée et remise au propriétaire à son adresse personnelle ${ }^{10}$. Or il est parfois très difficile d'identifier le propriétaire et les agences publiques se contentent le plus souvent d'afficher la notice sur le bâtiment sans contacter personnellement le propriétaire du bien. Cela rend la procédure engagée par la Ville illégitime devant la justice car tous les délais juridiques permettant au propriétaire d'intervenir lui-même ou de récuser l'intervention municipale courent à compter de la date de cette notification. Si le propriétaire n'a pas été dûment informé des mesures prises contre lui, le juge en charge de l'affaire peut décider l'abandon des poursuites pour vice de forme. Cette règle a pour effet de protéger les propriétaires car il incombe à la Ville - dont les moyens financiers sont limités - de trouver l'identité du propriétaire et son adresse actuelle.

Nombre de propriétaires sont difficiles à retrouver car les registres de la Ville n'ont pas été mis à jour au rythme du départ d'une partie des résidents depuis les années 1970, de sorte que les autorités ne disposent pas de l'adresse actuelle de tous les propriétaires ou de leurs ayants-droit. En outre, plus de $59 \%$ des propriétés concernées par des arriérés d'impôts appartiennent à des propriétaires qui ne résident pas sur place (Plan Philly, 9 mars 2013), voire qui résident hors de Pennsylvanie. Il est d'autant plus difficile pour la Ville de localiser les propriétaires concernés que certains d'entre eux recourent à des sociétés-écrans pour se rendre introuvables. Sur les registres de l'Office of Property Assessment (OPA), nombre de propriétés sont enregistrées au nom d'une compagnie, et l'adresse « hors site » du propriétaire - c'est-à-dire l'adresse à laquelle joindre le propriétaire non-occupant - correspond en fait à l'adresse de la propriété vacante. Une façon couramment employée de dénommer ce type de société consiste à utiliser l'adresse de la propriété, suivie par exemple des suffixes " LLC » ou «LP ». Un bref aperçu des noms utilisés à Kensington révèle l'ampleur de ce phénomène. Le site phillyaddress.com utilise les données fournies par le site internet de l'OPA mais avec la possibilité de lancer une recherche par nom de propriétaire plutôt que par adresse, ce qui permet d'afficher toutes les propriétés enregistrées au nom recherché. Une requête pour le nom "Frankford », c'està-dire l'une des principales avenues de Kensington, dévoilait en mai 2012 plus d'une vingtaine de propriétés enregistrées au nom de sociétés contenant le nom «Frankford » (1325 Frankford Associates, 2609 Frankford LLC, 4635 Frankford Avenue LP, etc.). C'est cette méthode qui a permis à Robert Coyle, surnommé le slumlord millionaire de Kensington, de bâtir durant plus de dix ans un empire de quelques centaines de propriétés en grande partie vacantes et mal entretenues sans être inquiété. Son acte d'accusation ${ }^{11}$ précise ainsi : Defendant Robert Coyle Sr., through various entities, owned and/or rented more than 300 properties in Philadelphia, mainly in low-income neighborhoods. The entities included, among others, LVJ LLC, Landvest LLP, Landvest LLP II, Landvest LLP III, Alivest LLP, Nine LLC, Ten Investments LLC, Memory LLC, Ralcram LLC, JC Real Estate

10 Cf. Philadelphia Property Maintenance Code, Chap. 3, Section PM-306.3

11 Disponible en ligne : http://media.philly.com/documents/coyle_indictment.pdf. 
Investments and Otay LLC. These entities were created by defendant Coyle for the purpose of, among other things, acquiring real property to hold for rental income and appreciation.

\subsection{Quand la loi fait désordre : une législation peu contraignante et mal appliquée}

Face à la rétention foncière et au maintien de propriétés dégradées, la Ville dispose de différents moyens d'action qui ne suffisent pas à endiguer le problème des propriétés dangereuses ou abandonnées.

Lorsqu'une propriété est définie comme tax-delinquent, c'est-à-dire que la taxe foncière est restée impayée durant plus de neuf mois, ou que le montant des taxes et des "liens" 12 impayés excède la valeur de la propriété, la propriété peut être mise aux enchères (Sheriff Sale) par le bureau du Sheriff et tout un chacun peut enchérir. C'est une autre échelle institutionnelle qui entre alors en jeu car le bureau du Sheriff dépend du comté, et non de la municipalité. Cette procédure est le plus souvent initiée par la Ville pour recouvrer l'ensemble des frais associés à une propriété : impôts impayés, mais aussi frais de démolition ou de sécurisation éventuels. À Philadelphie, le nombre considérable de propriétés concernées explique la lenteur des autorités à réagir. En effet, selon une enquête conjointe des journalistes de Plan Philly et du Philadelphia Inquirer (9 mars 2013), la ville comptait en avril 2011 près de 100000 propriétés appartenant à la catégorie tax-delinquent, soit presque $20 \%$ du nombre total de propriétés pour une dette équivalant à $10 \%$ des recettes fiscales que devrait rapporter la taxe foncière. La Ville n'est donc pas en mesure d'envoyer toutes les propriétés concernées au bureau du Sheriff à un rythme suffisant pour contenir le problème.

Dans tous les autres cas, la Ville n'a la possibilité d'agir que si la propriété est blighted, c'est-à-dire dans un état de dégradation qui menace la sécurité des habitants et déprécie la valeur des propriétés voisines. Entrent dans cette catégorie non seulement les propriétés mal entretenues (insalubres, dangereuses, présentant un risque d'incendie), mais aussi les propriétés "abandonnées ", c'est-à-dire inoccupées depuis plus d'un an et dont les taxes ou la dette à l'égard de la Ville n'ont pas été payées (Philadelphia Property Maintenance Code, Chap. 3, $P M-309.1$. Soit la propriété fait l'objet d'un projet immobilier et la Philadelphia Redevelopment Authority (PRA) $)^{13}$ peut alors la saisir via la procédure d'Eminent

12 Le terme " liens » désigne toutes les dettes associées à une propriété, et dont le montant est remboursé lors de la vente/saisie du bien si le propriétaire ne s'en acquitte pas.

13 Créée par la Urban Redevelopment Law de 1945, la PRA a pour objectif principal de remédier au délabrement urbain, et ce en faisant l'acquisition de propriétés vacantes ou en mauvais état et en veillant à leur réaménagement par un promoteur sous contrat. Elle dispose pour ce faire d'un financement par l'État fédéral et doit tirer des revenus supplémentaires de la vente de la propriété une fois la rénovation terminée. 
Domain $^{14}$ dans le but de la céder ensuite à un promoteur ; soit le bâtiment n'est pas entretenu mais aucun promoteur n'est intéressé, et il fera simplement l'objet d'une Violation Notice par le Department of Licenses and Inspection (L \& I) demandant les réparations nécessaires ou la démolition de l'édifice. La figure $n^{\circ} 5$ synthétise les différentes procédures possibles face à une propriété vacante.

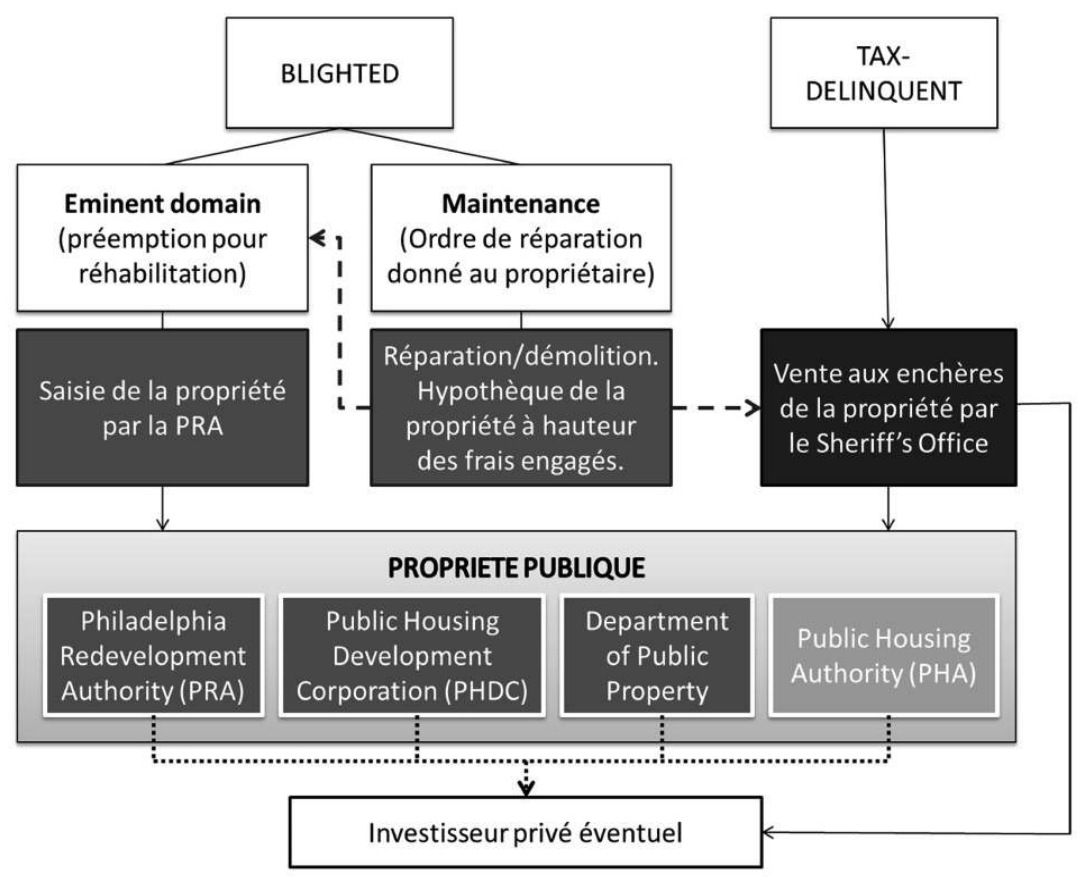

Echelon institutionnel concerné

Ville de Philadelphie $\square$ Comté de Philadelphie $\square$ Gouvernement fédéral

Fig. 5 Les différents recours contre une propriété vacante en fonction de son statut : un véritable parcours du combattant

The difficult course of action against vacant properties, depending on their status

Une fois le bien saisi par la ville, il incombe encore au promoteur ou au riverain qui souhaite l'acquérir d'effectuer les démarches relatives à l'acquisition d'une propriété publique, qui varient en fonction de l'institution qui a fait l'acquisition de la propriété. Toutes ces démarches suscitent l'intervention de nombreuses

14 La procédure d'Eminent Domain permet à une instance publique (en l'occurrence la Ville) de saisir une propriété privée pour cause d'utilité publique. Elle a été mise en place pour faciliter l'action de renouvellement urbain confiée à la PRA. 
instances différentes, ce qui rend le processus particulièrement laborieux pour les services municipaux comme pour d'éventuels investisseurs privés. Cela explique en grande partie le désintérêt des acheteurs privés pour ces propriétés publiques, d'autant que, en plus d'être complexe, le processus d'acquisition d'une propriété vacante est long : d'après le rapport de Kromer (2002), la saisie d'une propriété par Eminent Domain prend environ neuf mois, auxquels il faut ajouter trois mois à un an pour le transfert de la propriété à un promoteur par la PRA. Et ce délai peut atteindre huit à dix ans selon les personnes interrogées.

La législation relative aux transferts de propriété n'explique pas à elle seule le maintien de vastes terrains vagues et d'usines abandonnées dans les quartiers comme Kensington. Les modalités d'application de ces lois sont aussi un facteur d'inertie indéniable : les moyens financiers, techniques et humains manquent pour mettre en œuvre les procédures de gestion des propriétés abandonnées.

La première raison pour laquelle la Ville de Philadelphie est assez peu intervenue jusqu'ici contre les propriétaires en situation illégale réside dans l'impuissance de ses services à apporter une réponse ciblée à ce phénomène. Selon l'ancien directeur de l'OHCD, when cities were reorganized after WWII, there was no Department that was responsible for dealing with vacant properties [...]. So figuring out who in the City would be responsible for dealing with this has been a problem in every city, and it still hasn't been quite resolved. L'enchevêtrement des responsabilités dans ce domaine tend à entraver toute initiative. Pour faire l'acquisition d'une propriété publique, ne serait-ce qu'une parcelle vide, il faut déterminer quelle agence possède le terrain et suivre le processus d'acquisition propre à cette institution. Each agency has its own rules for how they manage [their land inventory]. So if you go to one agency, they might price things differently than another agency, they have different design standards, different qualifications... selon notre interlocutrice à la PRA. Les administrations successives ont avancé la volonté de réorganiser ce système pour le rendre plus efficace mais une telle réforme n'a toujours pas été menée à son terme.

Au-delà du manque de coordination des différentes structures publiques, l'inertie des autorités s'explique par un ensemble de dysfonctionnements majeurs au sein de la principale institution concernée : la Philadelphia Redevelopment Authority (PRA). Jusqu'à la réforme opérée en 2008, elle pouvait par exemple faire l'acquisition de propriétés sans l'accord du service financier. L'ancienne collaboratrice de la PRA que nous avons rencontrée évoque cette question sur un ton polémique : It was really like giving somebody a credit card and letting them going spend money, but without checking to see if there was money in the bank! So they ended up overspending 13 million dollars because nobody had to check with the finance people (entretien du 13 avril 2012).

D'autre part, l'institution ne disposait pas d'une liste répertoriant toutes les propriétés qu'elle possédait, et encore moins de cartes permettant de situer précisément chaque parcelle : The way the Redevelopment Authority tracked land acquisition was very backwards, it was literally on paper. There was no database 
that was centralized. [...] this City government literally didn't know what it owned before we came in in 2008 (ibid.).

Enfin, une fois la propriété confiée à un promoteur, la Redevelopment Authority est normalement chargée de s'assurer que le contrat présentant le projet d'aménagement a été honoré, et elle peut reprendre son bien si ce n'est pas le cas. Ce suivi, dernière étape de la mission assumée par la PRA, n'avait jamais été assuré en cinquante ans d'activité selon la personne que nous avons interrogée. Ces manquements ont été corrigés depuis 2008 si l'on en croit notre interlocutrice, mais il est encore trop tôt pour pouvoir apprécier l'effet de cette réforme sur la gestion du foncier par la PRA.

Le manque de réactivité de la Ville à l'égard des propriétaires qui ne respectent pas la loi tient en grande partie au manque de moyens dont souffrent la plupart des structures intervenant dans la gestion et le transfert de propriétés foncières. Les services du Department of Licenses and Inspection (L \& I) n'ont ni le temps ni les moyens financiers nécessaires pour surveiller activement tous les bâtiments mal entretenus, et dès lors, ils sont contraints de faire un "tri" dans leurs interventions en se concentrant sur les propriétés les plus dangereuses selon Kromer (2002, p 39). Lorsque les bâtiments sont dégradés mais ne menacent pas la sécurité publique, ils doivent se contenter d'accumuler des amendes car il serait trop coûteux d'intervenir. Le Philadelphia Inquirer en date du 17 mai 2012 signale par exemple une fabrique textile au nord de Kensington, à l'angle des rues Ruth et Somerset, sur laquelle se sont accumulés 844000 dollars de nuisance liens ${ }^{15}$ depuis 1992. Les interventions sont limitées aux cas les plus urgents, car la Ville est bien consciente qu'elle a peu de chances d'obtenir le remboursement des frais engagés pour la sécurisation ou la démolition de bâtiments visiblement négligés depuis longtemps.

Un autre moyen pour la ville de gérer les bâtiments vacants serait de faire leur acquisition. Mais cette possibilité est encore plus problématique sur le plan financier que la précédente. Tout d'abord, la procédure d'acquisition est en ellemême très coûteuse puisque s'ajoute à l'ensemble des frais juridiques la "juste compensation" que la PRA doit verser au propriétaire. En outre, les pouvoirs publics n'ont pas intérêt à faire l'acquisition d'un trop grand nombre de propriétés car il leur est déjà difficile de compenser ponctuellement l'entretien défectueux des propriétés privées. This would be so expensive to maintain, the City couldn't pay for the Police explique Richard Negrin, managing director de la ville de Philadelphie depuis juillet 2010, avant d'ajouter : I don't want the City to be the biggest slumlord in Philadelphia (émission du 17 avril 2012, "Radio Times ", WHYY Radio). En d'autres termes, posséder des propriétés sans être capable de les entretenir, c'est se comporter comme les slumlords donc ce ne peut être la politique municipale. L'impuissance de la municipalité face aux propriétés problématiques, que ce soit

15 Il s'agit des frais engagés par la Ville pour sécuriser, entretenir ou démolir un bâtiment lorsque celui-ci menace la sécurité publique, et dont la dette est attachée au titre de propriété jusqu'à son remboursement par le propriétaire des lieux. 
d'un point de vue fiscal ou parce qu'elles menacent la sécurité publique, explique ainsi en grande partie l'inertie de quartiers comme Kensington où ces propriétés sont nombreuses. La Ville se retrouve alors prise dans un cercle vicieux : elle manque de moyens pour lutter contre les propriétés qui dévalorisent certains quartiers, mais en n'obligeant pas les propriétaires négligents à payer les taxes foncières, elle voit son budget déjà limité privé des bénéfices fiscaux qui lui sont dus.

\section{Conclusion}

Au cours de cette exploration des causes possibles de l'inertie observée dans le quartier de Kensington, il est apparu que le maintien d'une forte proportion de propriétés vacantes et de bâtiments dégradés s'expliquait en partie par la stratégie attentiste des acteurs privés. Dans un contexte où la propriété foncière et immobilière impose des coûts de rétention très faibles - et d'autant plus faibles que la municipalité n'a pas les ressources nécessaires pour recouvrer certains de ces frais - l'inaction apparait comme le meilleur choix d'un point de vue économique. De plus, Kensington fait partie des anciens quartiers industriels dégradés où la période de déclin économique et démographique entamée dans les années 1960 a favorisé l'activité spéculative des slumlords. Ceux-ci, comme les «marchands de sommeil » en France, perpétuent l'existence d'un bâti détérioré par leur stratégie de minimisation des dépenses. À cela s'ajoute l'exploitation des failles de l'action municipale de façon à s'exonérer des coûts supposés incompressibles que sont les dépenses d'entretien minimal et l'imposition locale.

Face à cette situation, les autorités locales sont impuissantes pour des raisons à la fois institutionnelles et économiques. La désorganisation des services chargés de la gestion des propriétés vacantes et le manque de moyens comparés à l'ampleur du phénomène expliquent en grande partie la capacité d'action limitée de la Ville de Philadelphie à l'encontre des propriétaires qui ne respectent pas la loi. Cette politique de laissez-faire fondée sur des ressources limitées rappelle la pratique du planned shrinkage ${ }^{16}$. Il s'agit de l'abandon sélectif des quartiers les plus dégradés via le retrait des services municipaux (pompiers, police, poubelles), de façon à encourager le dépeuplement progressif de ces quartiers et concentrer les ressources limitées de la ville sur des espaces jugés plus «viables ». Dans la mesure où les municipalités américaines, notamment dans la Rust Belt, subissent aujourd'hui une crise des finances locales d'une ampleur comparable à celle des années 1970, il est possible que le principe du planned shrinkage guide en partie l'action publique, mais cette question mériterait à elle seule une étude approfondie.

16 Ce principe, que l'on pourrait traduire par " décroissance organisée ", a été théorisé par Roger Starr, chef du service logement de New York dans les années 1970. 
Finalement, l'étude des stratégies de contrôle de l'espace via la propriété à Kensington éclaire le fonctionnement de ces anciens quartiers industriels qui peuvent sembler abandonnés aux yeux d'un observateur extérieur, mais sont en réalité au cœur de nombreux enjeux politiques et économiques locaux. L'extension de l'analyse à d'autres types de quartiers permettra sans doute de mieux comprendre les dynamiques urbaines récentes, dans un contexte où la crise des subprimes a remis en avant le rôle des questions de propriété dans la trajectoire des espaces urbains.

\author{
Université Paris Diderot \\ UMR 8504 Géographie-Cités \\ 13 rue du Four \\ 75006 Paris \\ florence.nussbaum@univ-paris-diderot.fr
}

\title{
Bibliographie
}

Aalbers, M. B. (2006), "When the banks withdraw, slum landlords take over: The structuration of neighbourhood decline through redlining, drug dealing, speculation and immigrant exploitation », Urban Studies, vol. 43, n 7, p. 1061-1086.

Avila, E. et Rose, M. H. (2009), "Race, Culture, Politics, and Urban Renewal : An Introduction", Journal of Urban History, vol. 35, n 3, p. 335-347.

Beauregard, R. A. (2002), Voices of Decline: The Postwar Fate of US Cities, New York, Routledge, $320 \mathrm{p}$.

Beauregard, R. A. (2005), " The textures of property markets : downtown housing and office conversions in New York City ", Urban Studies, vol. 42, n 13, p. 2431-2445.

Berry, B. J. L. (1985), "Islands of Renewal in Seas of Decay », in : P. E. Peterson (Ed.), The New Urban Reality, Washington, Brookings Institution Press, 301 p.

Bradbury, K. L., Downs, A. et Small, K. A. (1982), Urban Decline and the Future of American Cities, Washington, Brookings Institution Press, 309 p.

Charney, I. (2001), "Three dimensions of capital switching within the real estate sector: A Canadian case study ", International Journal of Urban and Regional Research, vol. 25, n 4, p. 740-758.

Defilippis, J. (1997), «From a Public Re-Creation to Private Recreation : The Transformation of Public Space in South Street Seaport ", Journal of Urban Affairs, vol. 19, n 4, p. 405-417.

Doussard, M., Peck, J. et Theodore, N. (2009), "After deindustrialization : Uneven growth and economic inequality in "postindustrial" Chicago ", Economic Geography, vol. 85, n² 2, p. 183207.

Garreau, J. (1992), Edge City : Life on the New Frontier, New York, Anchor, 576 p.

Gotham, K. F. (2002), «Beyond Invasion and Succession : School Segregation, Real Estate Blockbusting, and the Political Economy of Neighborhood Racial Transition », City \& Community, vol. 1, n 1, p. 83-111.

Ham, M. van, Manley, D., Bailey, N., Simpson, L. et Maclennan, D. (2013), Understanding Neighbourhood Dynamics : New Insights for Neighbourhood Effects Research, Dordrecht, Springer Netherlands, $284 \mathrm{p}$.

Hanlon, B. (2008), « The Decline of Older, Inner Suburbs in Metropolitan America », Housing Policy Debate, vol. 19, n 3, p. 423-456. 
Hanlon, B. (2009), Once the American Dream : Inner-Ring Suburbs of the Metropolitan United States, Philadelphie, Temple University Press, $224 \mathrm{p}$.

Hanlon, B., Short, J. R. et Vicino, T. J. (2009), Cities and Suburbs : New Metropolitan Realities in the US, New York, Routledge, 304 p.

Harvey, D. (1985), The Urbanization of Capital : Studies in the History and Theory of Capitalist Urbanization, Baltimore, John Hopkins University Press, 239 p.

Hillier, A. E. (2003), "Redlining and the Home Owners' Loan Corporation », Journal of Urban History, vol. $29, \mathrm{n}^{\circ} 4$, p. 394-420.

Kimble, J. (2007), " Insuring Inequality: The Role of the Federal Housing Administration in the Urban Ghettoization of African Americans ", Law \& Social Inquiry, vol. 32, n² 2, p. 399-434.

Kraut, D. T. (1999), "Hanging out the no vacancy sign : eliminating the blight of vacant buildings from urban areas », New York University Law Review, vol. 74, n² 4, p. 1139-1177.

Lee, S. et Leigh, N. G. (2007), «Intrametropolitan Spatial Differentiation and Decline of Inner-Ring Suburbs A Comparison of Four U.S. Metropolitan Areas », Journal of Planning Education and Research, vol. 27, $\mathrm{n}^{\circ} 2$, p. 146-164.

Ley, D. (1996), The New Middle Class and the Remaking of the Central City, New York, Oxford University Press, 383 p.

Massey, D. S. et Denton, N. A. (1993), American apartheid: segregation and the making of the underclass, Cambridge, Harvard University Press, 304 p.

Mikelbank, B. A. (2011), " Neighborhood Déjà Vu : Classification in Metropolitan Cleveland, $1970-$ 2000 », Urban Geography, vol. 32, n³ 3, p. 317-333.

Orser, W. E. (1994), Blockbusting in Baltimore : The Edmondson Village Story, Lexington, University Press of Kentucky, 256 p.

Peterson, P. E. (2001), The New Urban Reality, Washington, Brookings Institution Press, 301 p.

Popelard, A. (2009), " Détroit, catastrophe du rêve », Hérodote, n 132, p. 202-215.

Rast, J. (2001), «Manufacturing Industrial Decline: The Politics of Economic Change in Chicago, 1955-1998 », Journal of Urban Affairs, vol. 23, n², p. 175-190.

Reese, L. A. (2006), «Economic Versus Natural Disasters : If Detroit Had a Hurricane... », Economic Development Quarterly, vol. 20, n³, p. 219-231.

Saegert, S., Fields, D. et Libman, K. (2009), « Deflating the Dream : Radical Risk and the Neoliberalization of Homeownership », Journal of Urban Affairs, vol. 31, n 3, p. 297-317.

Satter, B. (2010), Family Properties : How the Struggle Over Race and Real Estate Transformed Chicago and Urban America, New York, Picador, 528 p.

Seligman, A. I. (2005), Block by Block: Neighborhoods and Public Policy on Chicago's West Side, Chicago, University of Chicago Press, 320 p.

Smith, N. (1979), "Toward a Theory of Gentrification : A Back to the City Movement by Capital, not People ", Journal of the American Planning Association, vol. 45, $n^{\circ}$ 4, p. 538-548.

Squires, G. D. (2005), "Predatory Lending : Redlining in Reverse », Shelterforce Online, n 139. http://www.nhi.org/online/issues/139/redlining.html

Vicino, T. J. (2008), "The spatial transformation of first-tier suburbs, 1970 to 2000: The case of metropolitan Baltimore ", Housing Policy Debate, vol. 19, n³, p. 479-518.

Wilson, J. Q. et Kelling, G. L. (1982), "Broken windows : The Police and Neighborhood Safety », Atlantic monthly, $\mathrm{n}^{\circ} 249$, p. 29-38.

Wilson, W. J. (1997), When Work Disappears : The World of the New Urban Poor, New York, Vintage Books, $322 \mathrm{p}$. 
Wyly, E. K., Atia, M., Foxcroft, H., Hamme, D. J. et Phillips-Watts, K. (2006), " American home : Predatory mortgage capital and neighbourhood spaces of race and class exploitation in the United States », Geografiska Annaler: Series B, vol. 88, n 1, p. 105-132.

Wyly, E. K. et Hammel, D. J. (1999), "Islands of decay in seas of renewal : Housing policy and the resurgence of gentrification », Housing Policy Debate, vol. 10, n 4, p. 711-771.

Zukin, S. (2009), Naked City: The Death and Life of Authentic Urban Places, New York, Oxford University Press, 312 p.

\section{Rapports}

United States Government Accountability Office (GAO), Nov. 2011, Vacant Properties - Growing Number Increases Communities' Costs and Challenges, GAO-12-34, Report to the Ranking Member, Subcommittee on Regulatory Affairs, Stimulus Oversight, and Government Spending, Committee on Oversight and Government Reform, House of Representatives, 102 p.

Econsult Corporation, Penn Institute for Urban Research \& May 8 Consulting, Nov. 2010, Vacant Land Management in Philadelphia : The Costs of the Current System and the Benefits of Reform. Prepared for the Redevelopment Authority of the City of Philadelphia and the Philadelphia Association of Community Development Corporations, $32 \mathrm{p}$.

Philadelphia Local Initiatives Support Corporation (LISC), 2009, Improving Philadelphia's Vacant Property Programs. Prepared for the National Vacant Properties Campaign, 30 p.

Kromer, J., Oct. 2002, Vacant-property policy and practice : Baltimore and Philadelphia, Fels Institute of Government, University of Pennsylvania. Prepared for the Brookings Institution Center on Urban and Metropolitan Policy and CEOs for Cities, 62 p.

\section{Presse}

"A landlord dropped a bomb on Kensington. It could happen again », Isaiah Thompson, The Philadelphia City Paper, 9 juin 2010 - http://archives.citypaper.net/articles/2010/06/10/kensingtonphiladelphia-slumlord-robert-coyle

"Abandoned factories pose a problem in reviving Philly areas », Graham Troy, The Philadelphia Inquirer, 17 mai 2012 - http://articles.philly.com/2012-05-17/news/31727744_1_factories-disastrousfire-industrial-land.

"Philly's crumbling vacant buildings, \& what to do about them », émission du 17 avril 2012, Radio Times with Marty Moss-Coane, WHYY Radio. Invités : Richard Negrin, Patrick Kerkstra et Christopher Sawyer.

«Ravaged by Neglect - Part One : Lax property tax enforcement and brazen deadbeat investors have depressed Philadelphia's property-tax base by $\$ 9.5$ billions », Patrick Kerkstra, Plan Philly, 9 mars 2013 - http://planphilly.com/articles/2013/03/09/part-one-lax-property-tax-enforcementand-brazen-deadbeat-investors-have-depressed-philadelphia-s-property-tax-base-by-9-5-billion 